\title{
Volume Element Model Mesh Generation Strategy and its Application in Ship Thermal Analysis
}

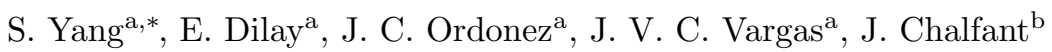 \\ ${ }^{a}$ Department of Mechanical Engineering, Energy and Sustainability Center, and Center for \\ Advanced Power Systems, Florida State University, Tallahassee, FL 32310, USA \\ ${ }^{b}$ MIT Sea Grant Design Laboratory, Massachusetts Institute of Technology, Cambridge, MA \\ 02139, USA
}

\begin{abstract}
This paper introduces a mesh generation strategy devised and implemented for the volume element model (VEM), and elaborates key contributions of the strategy in enhancing the VEM as a prominent tool in ship thermal modeling and simulation. The VEM mesh generation strategy employs ray crossings and ray-triangle intersection algorithms developed in previous studies, and constructs sufficiently accurate geometric representations of the whole ship within permissible time frame using hexahedral meshes. In addition, this work demonstrates the strategy's practicality in thermal analysis of a notional all-electric ship, which is characterized by intricate structures and multiple internal components, i.e., thermal loads. Ship thermal solutions obtained in this assessment verify the proposed mesh generation strategy's ability to improve the overall computational efficiency of the VEM, by allowing it to obtain plausible thermal solutions with respect to time and space using a coarse independent mesh.

Keywords: volume element model, ship mesh generation, ship thermal analysis, all-electric ships
\end{abstract}

\footnotetext{
*Corresponding author. Tel: +1-937-657-4156

Email addresses: syang@caps.fsu.edu (S. Yang), Emerson.dilay@up.com.br (E. Dilay), ordonez@caps.fsu.edu (J. C. Ordonez), jvargas@caps.fsu.edu (J. V. C. Vargas), chalfant@mit.edu (J. Chalfant)
}

Preprint submitted to Advances in Engineering Software

July 15, 2015

(C) 2015. This manuscript version is made available under the Elsevier user license http://www.elsevier.com/open-access/userlicense/1.0/ 


\section{Nomenclature}

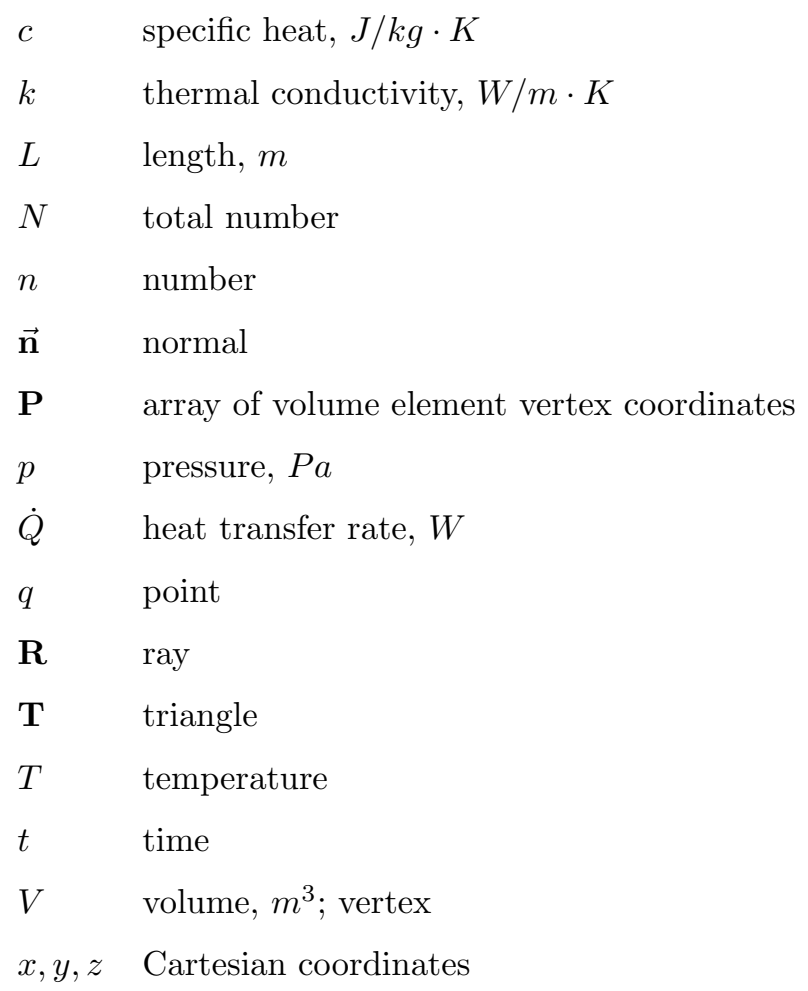




\begin{tabular}{|c|c|}
\hline$b$ & bottom \\
\hline div & divider \\
\hline$e$ & east \\
\hline$e q$ & equivalent \\
\hline gen & generation \\
\hline$i$ & VE index \\
\hline$j$ & VE face index \\
\hline $\max$ & maximum \\
\hline $\min$ & minimum \\
\hline$m$ & mesh \\
\hline$n$ & north \\
\hline$R$ & actual component weighted average \\
\hline$s$ & south \\
\hline$t$ & top \\
\hline$v$ & vapor \\
\hline$V E$ & volume element \\
\hline vs & saturation pressure \\
\hline$w$ & west \\
\hline$x$ & $\mathrm{x}$-direction \\
\hline$y$ & $\mathrm{y}$-direction \\
\hline$z$ & z-direction \\
\hline
\end{tabular}

\section{Introduction}

Volume Element Model (VEM) is a three-dimensional (3D) dynamic reducedorder model developed for its application in thermal systems engineering [1] 5 The scheme discretizes the domain of interest (i.e., system under analysis) in three dimensions using a cell-centered finite-volume scheme [2]. Subsequently, fundamental laws of thermodynamics and heat transfer are applied to each cell in conjunction with empirical correlations in order to quantify the energy transfer between cells. The resulting system of nonlinear ordinary differential equations 
(ODEs) with respect to time are solved using an appropriate numerical method according to the problem under consideration. Previously, the scheme has been employed to study thermal responses of several dynamic systems [1, 3, 4, and it has been experimentally validated using Power Electronic Building Blocks [5] and an Off-Grid Zero Emissions Building [6].

The mesh generation strategy devised and implemented into the VEM plays a key role in the enhancement of the overall VEM computational efficiency, defined as accuracy achieved per unit execution time 22. The proposed VEM mesh generation strategy creates a ship mesh composed of structured hexahedral elements within acceptable time frame, and it allows the VEM to obtain plausible numerical solutions using a coarse independent mesh. Furthermore, the strategy allows for the visualization and analysis of VEM computational grids and numerical results using VisIt visualization software [7.

In recent years, several studies have been published proposing methods to construct and refine hexahedral meshes [8] 17. Mesh generation techniques and algorithms developed in previous works created 3D meshes with great accuracy as a result of novel refinement techniques like the octree node-boundary adjustment. The technique presented by Ito et al. [13, for instance, is an effective mesh generation and refinement method which allows to construct computational grids of large and complex geometries with a small number of hexahedral so elements. In addition, Ruiz et al. [16] combined grid-based and advancing front methods to generate unstructured hexahedral meshes of exterior domains and reproduce the shape of distorted boundaries.

According to [18, hexahedral meshes are often preferred over other meshes (e.g., tetrahedral), when available, for a variety of reasons. Typically, tetrahedral meshes require 4-10 times more elements than a hexahedral mesh to obtain the same level of accuracy [19, 20]. Furthermore, in some types of numerical approximations (e.g., high deformation structural finite element analysis), tetrahedral elements are mathematically stiffer due to a reduced number of degrees of freedom associated with a tetrahedral element 21]. In their paper [18, Shep-

40 herd and Johnson also outline existing constraints of hexahedral meshes and 
proposes possible solutions to overcome such limitations.

Hexahedral meshes have been employed to solve a variety of problems ranging from CFD to metal forming processes [9 11, 17, 19, 21, 22, In [10, Kwak and Im studied the practicality of employing hexahedral elements for 3D metal

45 forming simulations. Based on their assessment using distributions of effective strain-rate graident and posteriori error values, the authors construed that the proposed hexahedral mesh could be effectively used to simulate metal formations. De Santis et al. [17] developed a full-hexahedral structured meshing technique for image-based computational vascular modeling. The authors indicated that in solving the Navier-Stokes equations in a left coronary artery, tetrahedral (unstructured) meshes needed much higher resolution than structured hexahedral meshes to reach mesh independency, with higher computational costs. Such differences were also reported in other studies and were attributed to the misalignment of meshes with the flow direction, causing high numerical diffusion error associated with unstructured meshes [22, 23].

Among numerous dynamic systems, ship thermal modeling and simulation, in particular, generally require an exorbitant amount of time to obtain meshindependent solutions due to large and complex ship geometry (e.g., curved surfaces) and multiple internal components. As a result, numerous ship mesh generation techniques have been presented previously [24 27] in efforts to expedite mesh construction and refinement processes for finite element method. These techniques, however, are restricted to finite element analysis which usually demands high computing power to generate mesh and simulate large systems such as ships. Therefore, the objective of this paper is to present the effort to apply hexahedral mesh generation techniques and algorithms developed previously [13, 28, 29] to the VEM. In addition, the practicality of the proposed mesh generation strategy in ship thermal modeling and simulation is discussed with an example problem. 


\section{Mesh generation strategy} tured mesh constituted of lumped control volumes, i.e., volume elements (VEs). The mesh generation strategy proposed in this paper consists of the following steps: (1) extraction of geometric information from a STL file; (2) generation of an enclosing mesh block that has sufficient size to comprise the entire CAD 75 geometry; and (3) intersection of the CAD geometry with the enclosing mesh block to produce the actual mesh. Furthermore, the strategy includes two additional steps in preparation for the numerical solver associated with the VEM: (i) Assignment of physical properties to each corresponding volume element in the computational domain; and (ii) writing output files for VisIt and the solver. with the VEM solver [30].

\subsection{Extraction of CAD geometry from the Stereolithography file}

The mesh generation process initiates by reading the geometric information from a STL file in ASCII format, then numbers each triangle and saves the coordinates of its vertex and unit normal vector into separate arrays. In addition, the algorithm reads an user-input text file including: the total desired number of VEs in $x, y$, and $z$ directions, number of vertical (e.g., walls, bulkheads, etc.) and horizontal (e.g., decks) dividers with predefined thicknesses, and spatial location, physical dimensions, as well as component (e.g., ship equipment) properties. Ships, for instance, feature bulkheads and decks to represent distinct compartments, zones, etc., and they often prevent the energy transfer between these spaces via heat and/or mass. VEs representing dividers may or may not have smaller volume depending on their predefined thickness. Assignment of multiple ship components within the mesh will further be discussed in Section $95 \quad 2.4$ 


\subsection{Hexahedral mesh block generation}

The next step in the VEM mesh generation strategy is to create an enclosing mesh block of hexahedral elements as shown in Figure 1 . The extreme values of $x, y$, and $z\left(x_{\min }, x_{\max }, y_{\min }, y_{\max }, z_{\min }\right.$, and $\left.z_{\max }\right)$ are identified in the array of vertices extracted from the STL file discussed in Section 2.1. Then the enclosing mesh block dimensions are determined as: $L_{x}=x_{\max }-x_{\min }, L_{y}=y_{\max }-y_{\min }$, and $L_{z}=z_{\max }-z_{\min }$ in the $x, y$ and $z$ directions, respectively.

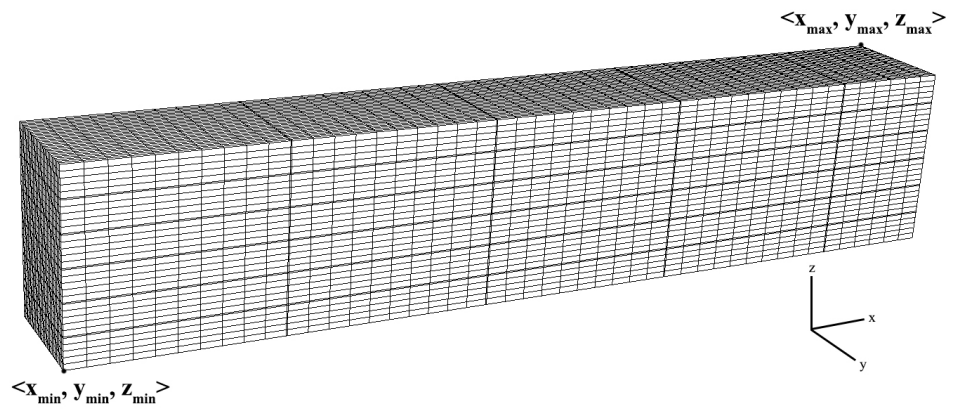

Figure 1: Enclosing mesh block consisting of 3D 8-node elements

In order to fit all VEs within the enclosing mesh block, the element size (i.e., volume) is computed based on $L_{x}, L_{y}, L_{z}$, the total number of elements in each respective spatial direction, and the total number of dividers with predefined thicknesses. The VE length in each spatial direction, $\delta_{x}, \delta_{y}$, and $\delta_{z}$, are computed as follows:

$$
\begin{aligned}
\delta_{x} & =\frac{L_{x}-\beta_{x} \cdot N_{d i v, x}}{N_{V E, x}-N_{d i v, x}} \\
\delta_{y} & =\frac{L_{y}-\beta_{y} \cdot N_{d i v, y}}{N_{V E, y}-N_{d i v, y}} \\
\delta_{z} & =\frac{L_{z}-\beta_{z} \cdot N_{d i v, z}}{N_{V E, z}-N_{d i v, z}}
\end{aligned}
$$

where $\beta_{x}, \beta_{y}$, and $\beta_{z}$ are divider thicknesses; $N_{d i v, x}, N_{d i v, y}$, and $N_{d i v, z}$ are the total number of dividers; and $N_{V E, x}, N_{V E, y}$, and $N_{V E, z}$ the total number of 
VEs; all in their respective spatial directions. In the light of the VEM solver, the number of VEs in each respective spatial direction is arbitrarily chosen for the first mesh, then it is successively refined until convergence is established according to the prescribed criterion for the problem under analysis. An example VEM mesh geometry is illustrated in Figure 2, where $N_{d i v, x}=N_{d i v, y}=N_{d i v},=$ 1 and $N_{V E, x}=N_{V E, y}=N_{V E, z}=3$.

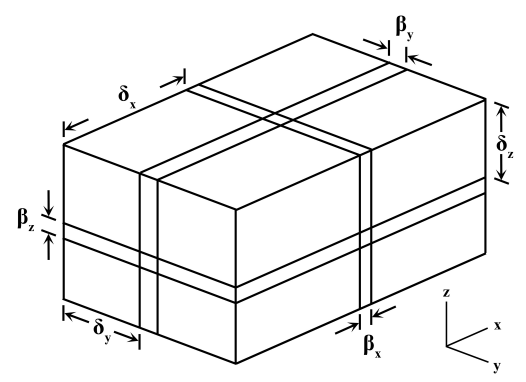

Figure 2: VEM mesh geometry where $\delta_{x}, \delta_{y}$, and $\delta_{z}$ are VE lengths and $\beta_{x}, \beta_{y}$, and $\beta_{z}$ are divider thicknesses, all in their respective spatial direction

Once its size (i.e., volume) is computed, VEs are constructed from the bottom left corner $<x_{\min }, y_{\min }, z_{\min }>$ (refer to Figure 1) in order to retain the coordinate information and the origin of the CAD geometry. Note that these minimum extreme coordinates are not necessarily the origin since these values may be negative instead of $\langle 0,0,0\rangle$. The algorithm then calculates the number of VEs between dividers $n_{d i v, i}$ according to the following equation:

$$
n_{d i v, i}=\frac{N_{V E, i}-N_{d i v, i}}{N_{d i v, i}+1}
$$

where $i=x, y$, or $z$. Since most physical systems have predefined number of dividers, this equation can be used to determine $N_{V E, i}$ based on the desired number of VEs between dividers. Such calculation also guarantees uniform divisions of volume elements in the mesh. 
Three loops are initiated to construct the enclosing mesh block with element length $\delta_{x}, \delta_{y}$, and $\delta_{z}$ for each respective spatial direction. Figure 3 illustrates the construction process for the mesh depicted in Figure 2 in a chronological order. In Figure $3 \mathrm{a}$, the lines are drawn from the $<x_{\min }, y_{\min }, z_{\min }>$ with lengths $\delta_{x}$ or $\beta_{x}$, depending on the number of VEs and dividers. $P_{x y z}$ is an array in which Cartesian coordinates of each VE vertex are saved according to its nodal point. In this example, there are two VEs in the $\mathrm{x}$-direction with a divider in the middle. If $<x_{\text {min }}, y_{\min }, z_{\min }>=<0,0,0>$, then $P_{111}=<0,0,0>$. Similarly, $P_{211}=<\delta_{x}, 0,0>, P_{311}=<\delta_{x}+\beta_{x}, 0,0>$, and $P_{411}=<2 \delta_{x}+\beta_{x}, 0,0>$. The same procedure is followed in the y-direction as shown in Figure $3 \mathrm{~b}$. Once the x-y plane is constructed, the algorithm repeats the same steps for $P_{x y 2}$ and so on as illustrated in Figure 35 and $3 \mathrm{~d}$. For instance, the coordinates of $P_{441}, P_{232}$, and $P_{113}$ are $\left.<2 \delta_{x}+\beta_{x}>,<2 \delta_{y}+\beta_{y}, 0\right\rangle,\left\langle\delta_{x}, \delta_{y}+\beta_{y}, \delta_{z}>\right.$, and $<0,0, \delta_{z}+\beta_{z}>$, respectively. Each loop ends when the counter equals the sum of the number of VEs and dividers in each spatial direction. In case non-uniform 130 divisions (i.e., an unequal number of VEs between dividers) are desired, the user can modify the algorithm to specify the number of VEs between dividers. 


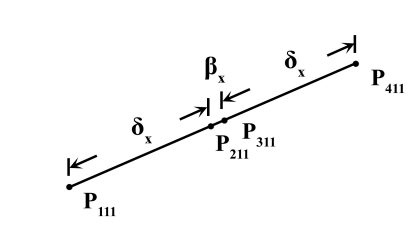

(a)

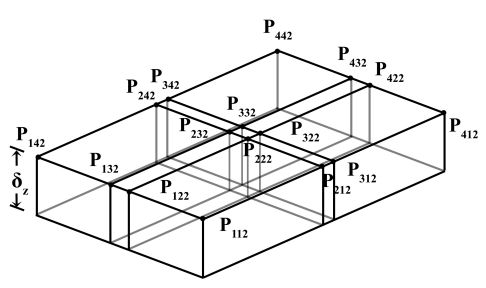

(c)

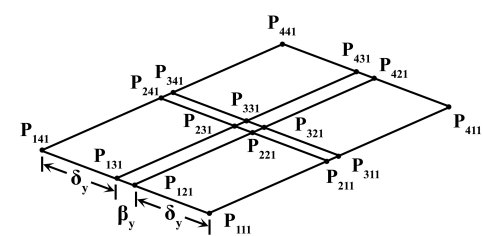

(b)

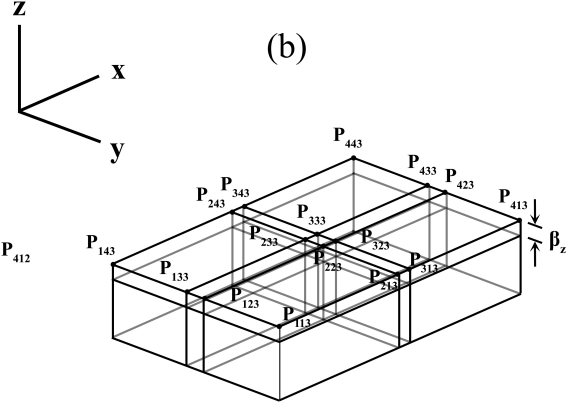

(d)

Figure 3: VEM enclosing mesh block generation steps: (a) a line representing two VEs and a divider of thickness $\beta_{x}$ is drawn from $\mathbf{P}_{111}$ in the x-direction with a total length of $2 \delta_{x}+\beta_{x}$; the same procedure is repeated in the $y$-direction (b), and in the z-direction (c) and (d)

Once the enclosing mesh block is generated, every VE is locally numbered with nodal points based on the right hand rule discussed in [28.

\subsection{Final mesh generation via search and intersection} etry in the enclosing mesh block as illustrated in Figure 4 must be eliminated. Previously, Lee and Yang [9] obtained the provisional mesh (final mesh) by taking grid points and cells of the superimposed uniform grid (enclosed mesh block) in the domain to be meshed as nodes and elements of the new mesh, respectively. In their work, a grid point was inside the domain of interest if it belonged to an element in the old mesh and was composed of six pyramids with positive volume. The elimination process in the proposed mesh generation strategy is performed by employing ray crossings and ray-triangle intersection algorithms [29, 31]. 


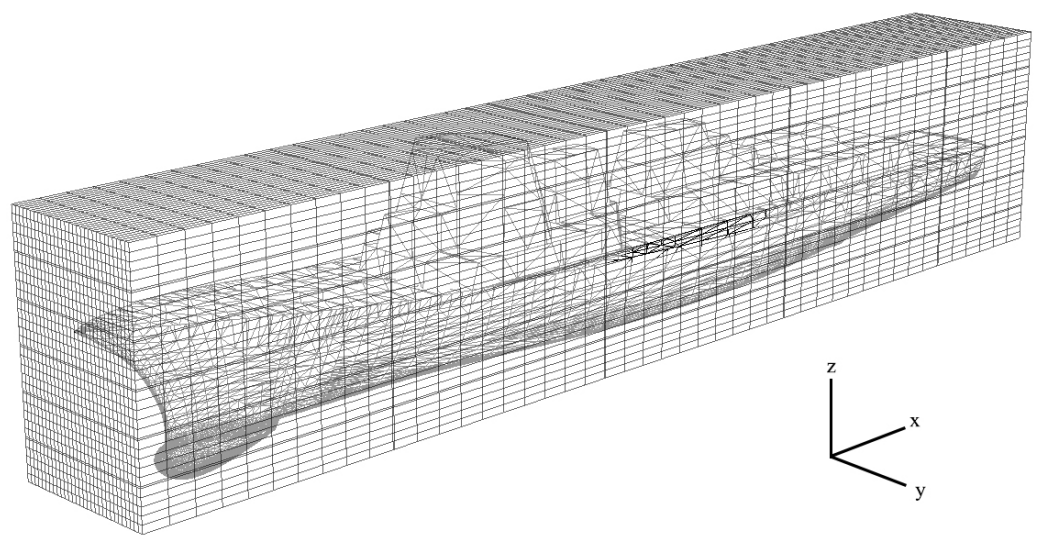

Figure 4: CAD geometry inside the enclosing mesh block

Ray crossings technique consists of drawing a ray from each mesh element center in an arbitrary direction and counting the number of intersections of the ray with the triangles. The element is located inside or outside of the CAD boundary if the number is odd or even, respectively. An example of ray crossings is elaborated with a 20-edge polygon depicted in Figure 5 where $q_{1}, q_{2}$, and $q_{3}$ are points from which rays $\mathbf{R}_{\mathbf{1}}, \mathbf{R}_{\mathbf{2}}$, and $\mathbf{R}_{\mathbf{3}}$ are drawn. According to the figure, $\mathbf{R}_{1}$ has an odd number of crossings while $\mathbf{R}_{\mathbf{2}}$ crosses the boundary twice; hence the point $q_{1}$ is inside and $q_{2}$ is outside of the domain. Special attention must be paid to any ray that hits a vertex, is collinear with an edge, or lies directly on the domain boundary; this is exemplified by $\mathbf{R}_{\mathbf{3}}$. In order to handle these specialcase intersections, the algorithm proposed in this paper applies the convention presented in [32, where for an edge to count as a crossing of a ray, one of the edge's endpoints must be strictly above the ray, and the other endpoint on or below. Based on this convection, edges $(4,5)$ and $(3,4)$ are not crossings while edges $(6,7),(11,12)$, and $(12,13)$ do count as crossings. Therefore $q_{3}$ is determined to lie inside the polygon.

In the ray-triangle intersection algorithm, objects are represented by vertices, edges, and faces; a segment by its endpoints; a ray as an extended segment; and a triangle by its three vertices. A parametric equation, $P(r)=P_{0}+r\left(P_{1}-P_{0}\right)$, 


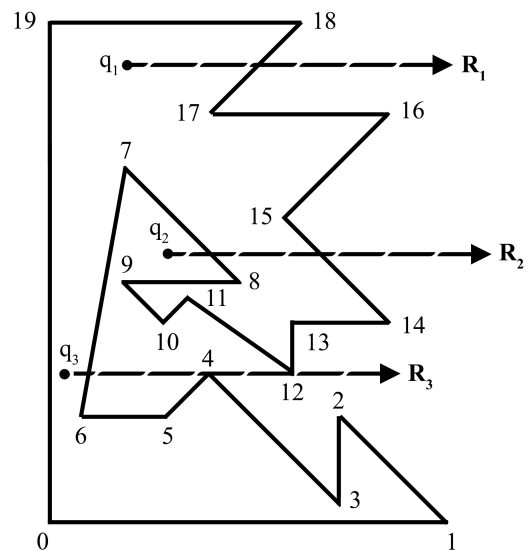

Figure 5: Rays crossing a 20-edge polygon where $q_{1}, q_{2}$, and $q_{3}$ have rays $\mathbf{R}_{\mathbf{1}}, \mathbf{R}_{\mathbf{2}}$, and $\mathbf{R}_{\mathbf{3}}$ drawn to their right.

is assigned to each line and used for the ray starting at $P_{0}$ going in the direction $\left(P_{1}-P_{0}\right)$, and $0 \leq r \leq 1$. In Figure 6 , a plane $\pi$ through the point $V_{0}$ with a normal vector $\tilde{\mathbf{n}}$ crosses the parametric line at the point $P_{i}=P\left(r_{i}\right)$ defined by the following parametric value [31]:

$$
r_{i}=\frac{\tilde{\mathbf{n}} \cdot\left(V_{0}-P_{0}\right)}{\tilde{\mathbf{n}} \cdot\left(P_{1}-P_{0}\right)}
$$

In Figure 6, consider a ray $\mathbf{R}$ from $P_{0}$ to $P_{1}$, and a triangle $\mathbf{T}$ with vertices $V_{0}, V_{1}$ and $V_{2}$ that lies in the plane $\pi$ through $V_{0}$ with the normal vector, $\tilde{\mathbf{n}}=$ $\left(V_{1}-V_{0}\right) \times\left(V_{2}-V_{0}\right)$. The intersection of $\mathbf{R}$ and $\pi$ is determined first to

verify if $\mathbf{R}$ lies in the plane, in which case the corresponding VE is eliminated.

Furthermore, $\mathbf{R}$ does not cross $\mathbf{T}$ if $\mathbf{R}$ and $\pi$ do not intersect, but if $\mathbf{R}$ and $\pi$ intersect at point $P_{i}=P\left(r_{i}\right)$, it is necessary to determine whether this point lies inside $\mathbf{T}$ or not.

Several techniques to test for the inclusion of a point inside a 3D planar triangle are available. The mesh generation strategy presented in this paper employs the method proposed and written by Sunday [31, which uses direct $3 \mathrm{D}$ computations to determine inclusion, avoiding the projection onto a $2 \mathrm{D}$ 


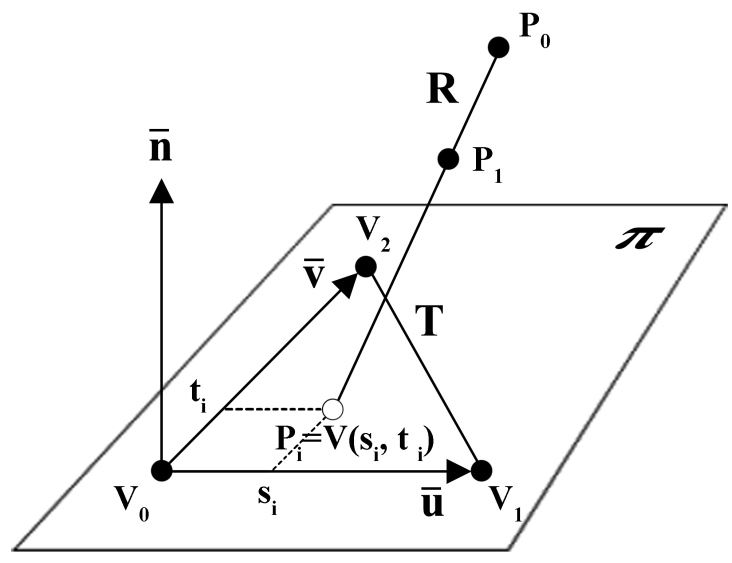

Figure 6: Illustration of ray-triangle intersection

coordinate plane. In this technique, a barycentric coordinate system for point $\mathrm{V}$ in the triangle $\mathbf{T}$ is defined as:

$$
V(s, t)=V_{0}+s\left(V_{1}-V_{0}\right)+t\left(V_{2}-V_{0}\right)=V_{0}+s \tilde{\mathbf{u}}+t \tilde{\mathbf{v}}
$$

where $\mathrm{s}$ and $\mathrm{t}$ are barycentric coordinates of $\mathrm{V}$ and $\tilde{\mathbf{u}}=\left(V_{1}-V_{0}\right)$ and $\tilde{\mathbf{v}}=$ $\left(V_{2}-V_{0}\right)$ are edge vectors of $\mathbf{T}$. A point $P=V(s, t)$ is inside $\mathbf{T}$ if and only if $s \geq 0, t \geq 0$, and $s+t \leq 1$. Hence for a given $P_{i}$, it is only required to find the weights $\left(s_{i}, t_{i}\right)$ and check these inequalities to verify its inclusion in $\mathbf{T}$. Barycentric coordinates are computed as the following:

$$
\begin{aligned}
& s=\frac{\tilde{\mathbf{w}} \cdot(\tilde{\mathbf{n}} \times \tilde{\mathbf{v}})}{\tilde{\mathbf{u}} \cdot(\tilde{\mathbf{n}} \times \tilde{\mathbf{v}})} \\
& t=\frac{\tilde{\mathbf{w}} \cdot(\tilde{\mathbf{n}} \times \tilde{\mathbf{u}})}{\tilde{\mathbf{v}} \cdot(\tilde{\mathbf{n}} \times \tilde{\mathbf{u}})}
\end{aligned}
$$

where $\tilde{\mathbf{w}}=P_{1}-V_{0}$ is another vector in $\pi$ described in [31. Three triangle vertices are given by: $V_{0}=V(0,0), V_{1}=V(1,0)$, and $V_{2}=V(0,1)$, and the point $P=V(s, t)$ is on an edge of $\mathbf{T}$ if one of these three conditions, $s=0$, $t=0$, or $s+t=1$, is satisfied (each condition corresponds to one edge). 
In the VEM mesh generation strategy, a ray $\mathbf{R}$ is drawn from each element center in the positive y-direction and the number of triangles that are crossed by the ray is recorded. Subsequently, the element is inside the CAD geometry if the number is odd, and outside otherwise, as mentioned previously. This part of the algorithm has the greatest cost in terms of computational time due to large calculations. Crossing a block of 30,000 VEs with 30,000 triangle boundaries, for example, requires a total of $30,000^{2}$ calculations to obtain the final mesh.

Once the elements outside of the CAD boundary are located and eliminated, all remaining VEs are renumbered using the right hand rule discussed at the end of Section 2.2. The number of VEs representing dividers (e.g., decks, bulkheads, etc.) are counted by searching for VEs with smaller length, width, or height depending on the direction in which the divider is assigned.

\subsection{Component and volume element property allocation}

Geometry meshing is now complete; however, additional steps are performed to facilitate the integration of the generated mesh with the VEM solver. In ship modeling, the domain of interest is generally constituted by several components (e.g., bulkheads, decks, equipment, etc.), compartment air, and cooling water.

Hence in the VEM, every VE represents a control volume that consists of a bounding box containing a fluid, solid, or mixture (solid+fluid), where each solid component is placed in one or more VEs according to their physical dimensions.

\subsubsection{Element allocation to a given component}

The component allocation process starts by identifying the vertex of the component with smallest $x, y$, and $z$ coordinates, to which component length $(d x)$, width $(d y)$, and height $(d z)$ are added to determine the other seven vertices. The eight resulting vertex coordinates are then compared with the nodal coordinates of all grid elements to determine the VEs to which the component is allocated with respect to the origin. In case the component size is smaller than a single VE, one element is allocated to ensure the component's presence. The 
algorithm calculates the number of VEs assigned to each equipment to verify if every ship equipment is included in the mesh.

\subsubsection{Adjacent elements}

In order to quantify the energy transfer between cells as depicted in Figure 7 the mesh generation algorithm searches for the neighboring element type of each VE at its north, south, east, west, top and bottom faces, and identifies it according to the three possible element types described previously: fluid, solid, or mixture (fluid+solid). All VEs contain fluid (i.e., air) by default unless solid or mixture element types are specified. The following interactions and boundaries are possible in the VEM computational domain: (1) fluid-fluid; (2) fluidmixture; (3) fluid-solid; (4) solid-solid; (5) solid-mixture; (6) mixture-mixture; (7) solid-domain boundary; (8) fluid-domain boundary; and (9) mixture-domain boundary. The domain boundary in the VEM mesh is surrounded by a virtual wall that is modeled using thermal resistance network, which accounts for heat transfers via convection between ship interior and exterior and the wall, conduction through the wall, and radiation between the ambient and the wall.

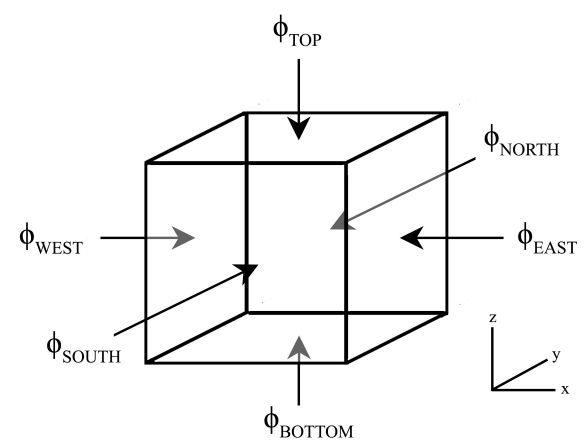

Figure 7: Possible energy transfer in a volume element through its north, south, west, east, top, and bottom faces

The VEM mesh generation strategy assigns physical properties (e.g., density, specific heat, thermal conductivity, heat generation rate, etc.) of every element type and component to corresponding VEs. Two possible methods are 
220

. Treat VE contents as a homogenous mixture of entities and calculate uniform properties for the $\mathrm{VE}$ using a weighted average proportional to the mass of each entity, or

2. Treat VE contents as a group of distinct entities, and separately apply the differential equation to each.

The second alternative requires more differential equations to be solved, therefore increasing the overall computational time. As a result, the first alternative is employed for the current VEM mesh generation technique, where equivalent densities $\left(\rho_{e q}\right)$ and thermal conductivities $\left(k_{e q}\right)$ for components are estimated as follows:

$$
\begin{array}{r}
\rho_{e q}=\frac{V_{R}}{V_{m}} \rho_{R} \\
k_{e q}=\left(\frac{V_{R}}{V_{m}}\right)^{\frac{1}{3}} k_{R}
\end{array}
$$

where $V_{R} / V_{m}$ is the ratio between the component physical and mesh volumes and $\rho_{R}$ and $k_{R}$ are the actual component weighted average density and thermal conductivity, respectively.

\subsubsection{Visualization of system components}

Visualization of the solid component distribution within the VEM computational grid is achieved by assigning specific scalar value to each solid component. VEs containing air, for instance, have a scalar value of zero while those representing solids have non-zero values. VisIt reads these values for its scalar mapping feature [7, where only elements with non-zero scalar values are shown in opaque colors. The output format can easily be modified to export results to alternative visualization software such as Paraview [33] and Mayavi 34. 


\subsubsection{Output files}

The VEM mesh generation strategy described above creates three output $T_{i}$ is the temperature of $\mathrm{VE} i, \rho$ is the density of the material inside the VE (fluid and/or solid), $V$ is the total VE volume, $c$ is either the specific heat of the solid/liquid or the specific heat at constant volume of the gas inside the VE $\left(c_{v}\right)$, $\dot{Q}_{e}, \dot{Q}_{w}, \dot{Q}_{t}, \dot{Q}_{b}, \dot{Q}_{n}, \dot{Q}_{s}$ are the heat transfer rates across the east, west, top, 

in [1].

The system of $N$ nonlinear ordinary differential equations defined by Eq. (7) formulates an initial value problem to be solved with a given initial condition $T_{i 0}$. Relative humidity of each VE that contains air is computed based on its temperature and assuming an initial relative humidity $\varphi_{i 0}$ :

$$
p_{v, i}=\varphi_{i 0} \cdot p_{v s}\left(T_{i 0}\right)
$$

where $p_{v, i}$ is the partial vapor pressure and $p_{v s}\left(T_{i 0}\right)$ is the water saturation pressure at $T_{i 0}$. Assuming constant absolute humidity throughout the simulation, the relative humidity $\varphi_{i}$ is computed as:

$$
\varphi_{i}=\frac{p_{v, i}}{p_{v s}\left(T_{i}\right)}
$$

where $p_{v s}\left(T_{i}\right)$ is the water saturation pressure at $T_{i}$. Solid VEs have zero relative humidity, i.e., $\varphi_{i}=0$.

\subsection{Numerical method}

270

The numerical method and its parameters are determined according to the numerical stability and desired solution accuracy for the problem under consideration. The VEM features several numerical schemes with adaptive time step size $\Delta t$ to solve for transient solutions, e.g., Backward Euler, Runge-KuttaFehlberg, Adams-Moulton, Gear's method, etc. If steady-state response is desired, the time derivative term on the left side of Eq. (7) is neglected and the resulting system of nonlinear algebraic equations is solved using Newton-Raphson method. In this ship thermal analysis, $4^{\text {th }}$ order Runge-Kutta method with adaptive $\Delta t$ was employed to solve the system of nonlinear ODEs with respect to time. 
The numerical convergence was verified by successive mesh refinements [35] and monitoring the variation in the Euclidean norm of the solution vector for the entire domain. The solution from a less refined mesh (mesh 1) were compared to the solution from a more refined mesh (mesh 2), and the refinement process stopped when the prescribed mesh refinement relative error, $\varepsilon_{m e s h}$, criterion was satisfied. Then mesh 1 was selected as the independent mesh based on the following equation:

$$
\varepsilon_{m e s h}=\frac{||\left|\Psi\left\|_{m e s h 1}-|| \Psi\right\|_{m e s h 2}\right|}{\|\Psi\|_{m e s h 1}} \leq 1 \times 10^{-3}
$$

280 computer with the following technical specifications: Core i5-2320 $3.0 \mathrm{GHz}, 8$ GB RAM, and Windows 7 64-bit operating system.

\subsection{All-electric ship mesh}

The notional all-electric ship hull similar to a DDG-51 destroyer is depicted in Figure 8 Table 1 lists ship dimensions and the number of VEs specified in each spatial direction for the independent ship mesh. The ship has five decks and four bulkheads in the $\mathrm{z}$ and $\mathrm{x}$-directions, respectively, with predefined thicknesses of $0.2 \mathrm{~m}$. In addition, there are 43 thermal loads (equipment) inside the ship and their physical locations and dimensions are listed in Table 2 For this mesh, uniform divisions were imposed between bulkheads and decks, i.e., there were an equal number of VEs between decks and bulkheads.

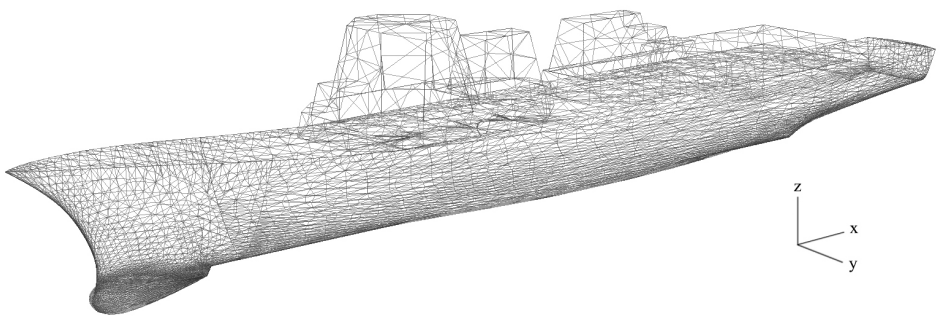

Figure 8: CAD drawing of a notional all-electric ship similar to a DDG-51 
Table 1: Notional all-electric ship dimensions and independent VE mesh size

\begin{tabular}{ccc}
\hline Direction & Length $(\mathrm{m})$ & $N_{V E}$ \\
\hline Bow to stern & 154 & 65 \\
Port to starboard & 20.9 & 31 \\
Keel to superstructure top & 29.1 & 50 \\
\hline
\end{tabular}

Comparisons between the VEM mesh and the actual ship geometry are illustrated in Figure 9, 10, and 11, with three meshes of different VE sizes as defined in Table 3 . According to these figures, more accurate representations of the ship were obtained as the number of VEs increased. Figure 11 depicts the top view of the ship superstructure, where the triangular region is not meticulously portrayed with coarse and fine meshes. Triangular geometries are difficult to represent with the VEM mesh due to their curved edges, which are difficult to construct with hexahedral elements unless smaller VE sizes are used.

Missing VEs near the ship boundary is due to the nature of ray-crossings and ray-triangle intersection techniques. If a large VE size (i.e., small $N_{V E, i}$ ) is selected, for instance, more than half of the VE, including its center, may be located outside of the ship boundary, leading the ray $\mathbf{R}$ to cross even number of triangles and consequently, eliminating the element from the final mesh. Although highly accurate representations of the CAD geometry were obtained by increasing the total number of VEs, CPU time required to generate the mesh concurrently increased as indicated in Table 3.

In order to approximate curved and distorted boundaries, Lee and Yang [9] used boundary fitting operation, in which the nodes on the zigzag boundary of the provisional mesh were projected on the boundary of the domain of interest. Subsequently, the layers of elements on the boundary surface (SEL) were constructed without thickness and the nodes were re-positioned by mesh smoothing to improve the shape. Accurate geometric representation, however, do not necessarily guarantee highly accurate numerical solutions, and such an effort often retards the mesh generation time without significant improvement 
Table 2: Notional all-electric ship component position and dimensions

\begin{tabular}{|c|c|c|c|c|c|c|}
\hline \multirow{2}{*}{ Component } & \multicolumn{3}{|c|}{ Position $(m)$} & \multicolumn{3}{|c|}{ Dimensions $(m)$} \\
\hline & $x$ & $y$ & $z$ & $d x$ & $d y$ & $d z$ \\
\hline SWHX_ZONE-1 & 27 & 1.7 & 0.2 & 5 & 2 & 2 \\
\hline CWU_ZONE-1 & 27 & -2.3 & 0.2 & 5 & 2 & 2 \\
\hline Load2_ZONE-1 & 20 & 1.7 & 9 & 5 & 2 & 2 \\
\hline Load3_ZONE-1 & 27 & 1.7 & 6 & 5 & 2 & 2 \\
\hline Load1_ZONE-1 & 20 & 1.7 & 6 & 5 & 2 & 2 \\
\hline SWHX_ZONE-2 & 50 & 0 & 0.2 & 5 & 3 & 2 \\
\hline CWU_ZONE-2 & 50 & -6 & 0.2 & 5 & 2 & 2 \\
\hline STBD_M_DCS_ZONE-2 & 50 & 6 & 9 & 5 & 3 & 2 \\
\hline STBD_M_ZONE-2 & 50 & 0 & 9 & 5 & 3 & 2 \\
\hline Load2_ZONE-2 & 50 & -6 & 12 & 5 & 3 & 2 \\
\hline Load3_ZONE-2 & 50 & 0 & 4 & 5 & 3 & 2 \\
\hline Load1_ZONE-2 & 50 & -6 & 4 & 5 & 3 & 2 \\
\hline MTG2_ZONE-2 & 50 & -6 & 9 & 5 & 2 & 2 \\
\hline PCM_AC-DC_ZONE-2 & 50 & 6 & 4 & 5 & 2 & 2 \\
\hline PLDCS_ZONE-2 & 60 & 0 & 12 & 5 & 3 & 2 \\
\hline Pulse_Load_ZONE-2 & 50 & 0 & 12 & 5 & 3 & 2 \\
\hline STBD_MD_DCS_ZONE-2 & 50 & 3 & 0.2 & 5 & 3 & 2 \\
\hline STBD_MD_ZONE-2 & 60 & 0 & 0.2 & 5 & 3 & 2 \\
\hline SWHX_ZONE-3-5 & 70.5 & 1.8 & 0.2 & 5 & 3 & 2 \\
\hline CWU_ZONE-3-5 & 70.5 & -4.2 & 0.2 & 5 & 2 & 2 \\
\hline MTG1_ZONE-3 & 100.5 & -4.2 & 0.2 & 5 & 2 & 2 \\
\hline PCM_AC-DC-1_ZONE-3 & 93.5 & 7.8 & 9 & 5 & 3 & 2 \\
\hline ATG1_ZONE-3 & 93.5 & 1.8 & 9 & 5 & 3 & 2 \\
\hline PCM_AC-DC-2_ZONE-3 & 93.5 & 4.8 & 0.2 & 5 & 2 & 2 \\
\hline Port-Motor-Drive_ZONE-3 & 93.5 & -4.2 & 9 & 5 & 3 & 2 \\
\hline Port_Motor_ZONE-3 & 93.5 & -4.2 & 4 & 5 & 3 & 2 \\
\hline Load2_ZONE-3 & 102 & 1.8 & 14 & 5 & 3 & 2 \\
\hline Load3_ZONE-3 & 93.5 & 1.8 & 4 & 5 & 3 & 2 \\
\hline Load1_ZONE-3 & 102 & 1.8 & 0.2 & 5 & 3 & 2 \\
\hline Radar_PCM-DC-DC-1_ZONE-5 & 65 & 1.8 & 14 & 3 & 2 & 2 \\
\hline Radar_ZONE-5 & 65 & -2 & 17 & 3 & 2 & 2 \\
\hline Radar_PCM-DC-DC-2_ZONE-5 & 65 & 2 & 17 & 3 & 2 & 2 \\
\hline Load3_ZONE-5 & 65 & 1 & 19 & 3 & 2 & 2 \\
\hline Load2_ZONE-5 & 75 & 1 & 19 & 3 & 2 & 2 \\
\hline Load1_ZONE-5 & 68 & 0 & 14 & 3 & 2 & 2 \\
\hline SWHX_ZONE-4 & 110 & 1.8 & 0.2 & 5 & 3 & 2 \\
\hline CWU_ZONE-4 & 110 & -4.2 & 0.2 & 5 & 2 & 2 \\
\hline PCM_AC-DC_ZONE-4 & 110 & 7.8 & 5.2 & 5 & 2 & 2 \\
\hline ATG2_ZONE-4 & 130 & 1.8 & 4 & 5 & 2 & 2 \\
\hline ESPCM_DC-DC_ZONE-4 & 130 & 5.8 & 6 & 5 & 2 & 2 \\
\hline Load3_ZONE-4 & 110 & -4.2 & 11 & 5 & 3 & 2 \\
\hline Load2_ZONE-4 & 120 & 7.8 & 8 & 5 & 3 & 2 \\
\hline Load1_ZONE-4 & 120 & -4.2 & 8 & 5 & 3 & 2 \\
\hline
\end{tabular}




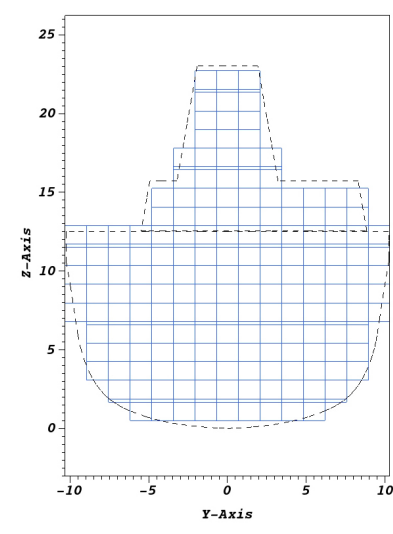

(a) Coarse

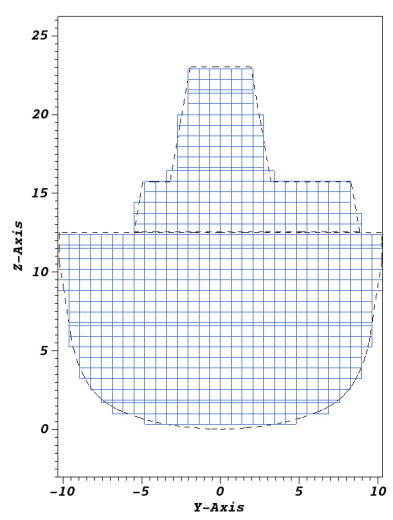

(b) Fine

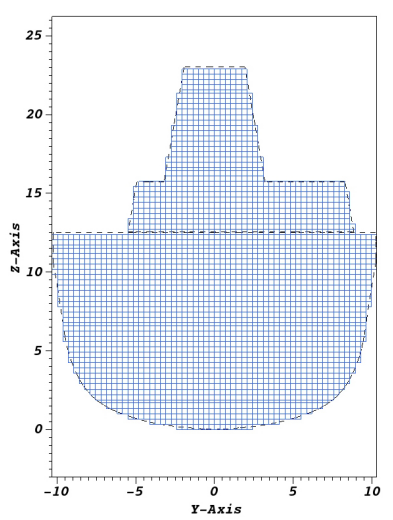

(c) Finest

Figure 9: Comparison of the ship cross-section in the y-z plane

in the solution accuracy, e.g., system-level simulations [1, 5].

Local mesh refinement methods allow for a detailed assessment of regions that are subject to delicate perturbations by adapting the precision of the numerical computation and refining the computational grids in and around those regions [36. Local mesh refinement strategies for hexahedral meshes have been presented in [10, 13] by implementing octree-based hexahedral mesh refinement scheme [8] with some modifications. This refinement technique can be applied to the proposed VEM mesh generation strategy by slicing the VE into several structured hexahedral elements (sub-VEs) as illustrated in [10, then assigning sub-indices and coordinates (i.e., $P_{x y z}$ ) to the vertices of each corresponding sub-VE. Subsequently, adjacent elements (i.e., boundaries) must be re-identified according to Section 2.4 .2 and appropriate equations can be applied.

In most system-level simulations such as that of ship, local mesh refinement is not as critical owing to significantly large systems' scale relative to their constituents. In addition, if the simulation objective is to quickly approximate dynamic system behavior during early design stages, local details may be neglected until meticulous analysis is required.

The independent ship mesh in Figure 12 was obtained in 259.46 CPU seconds 


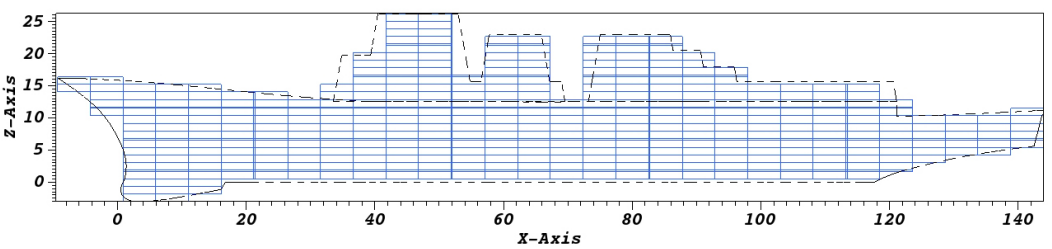

(a) Coarse

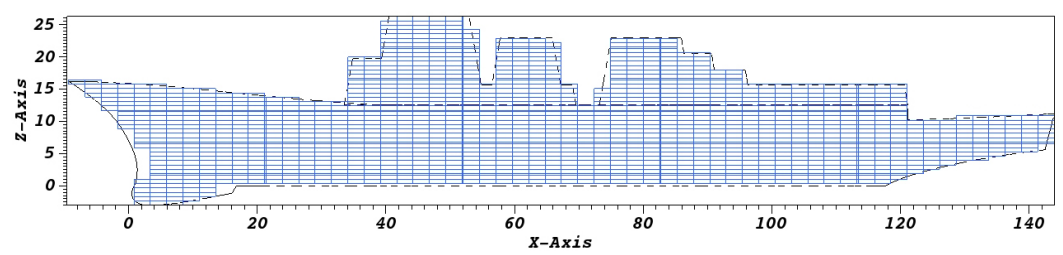

(b) Fine

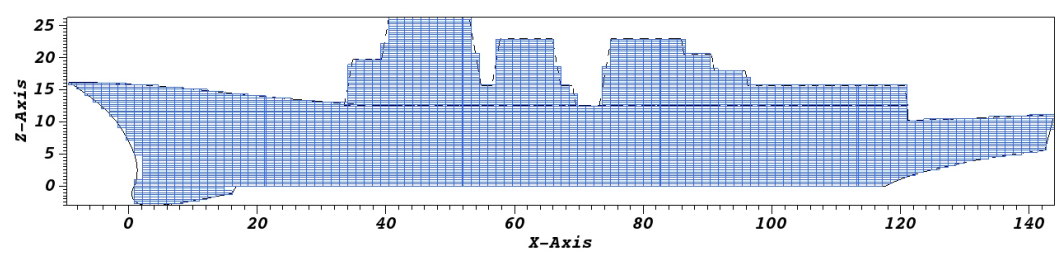

(c) Finest

Figure 10: Comparison of the ship cross-section in the x-z plane

and it consisted of 33,364 VEs, from which 4,407 VEs represented bulkheads and decks. Figure 13 depicts component distribution within the notional allelectric ship comprising 43 equipment, four bulkheads, and five decks, in which all solid components have the same scalar value of 1 (red).

The simulation required $600.41 \mathrm{CPU}$ seconds to solve the initial value problem and to evaluate the ship thermal response for 7,200 real-time seconds. Material properties and heat generation of each load included in this simulation are listed in [30. Ship temperature and relative humidity fields at $7,200 \mathrm{~s}$ are depicted in Figure 14 and 15 , respectively.

In Figure 14, ship boundary temperature and relative humidity fields at 7,200 s are shown. In Figure 14a ship substructure temperature is relatively lower since part of it is in the water at $280 \mathrm{~K}$. In addition, solar irradiation 


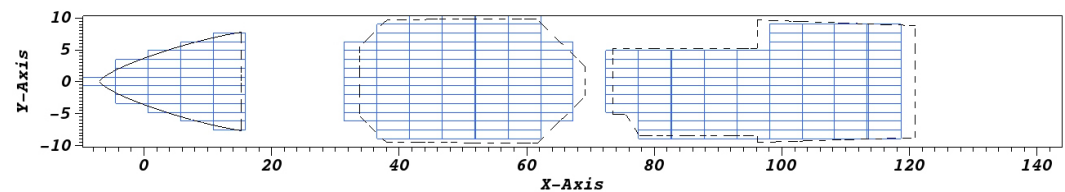

(a) Coarse

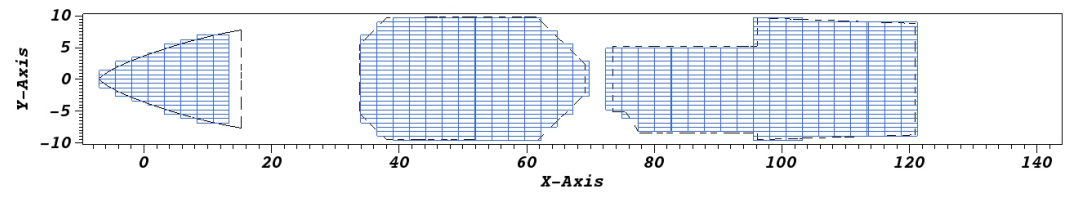

(b) Fine

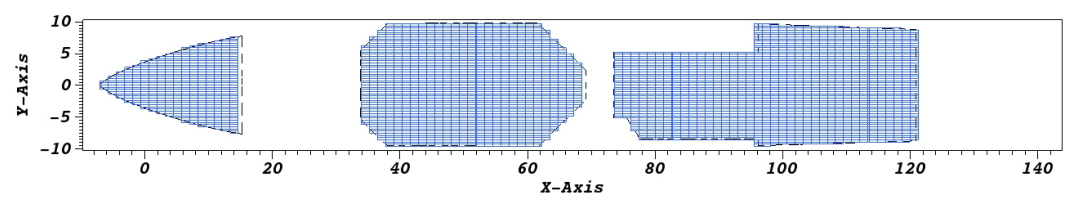

(c) Finest

Figure 11: Comparison of the ship cross-section in the x-y plane

Table 3: VE meshes of different sizes and their processing times

\begin{tabular}{cccccc}
\hline Mesh & $N_{V E, x}$ & $N_{V E, y}$ & $N_{V E, z}$ & $N_{V E, \text { final }}$ & CPU time (s) \\
\hline Coarse & 34 & 15 & 29 & 5,253 & 30.41 \\
Fine & 64 & 30 & 47 & 31,499 & 234.37 \\
Finest & 124 & 60 & 89 & 227,251 & $1,825.25$ \\
\hline
\end{tabular}

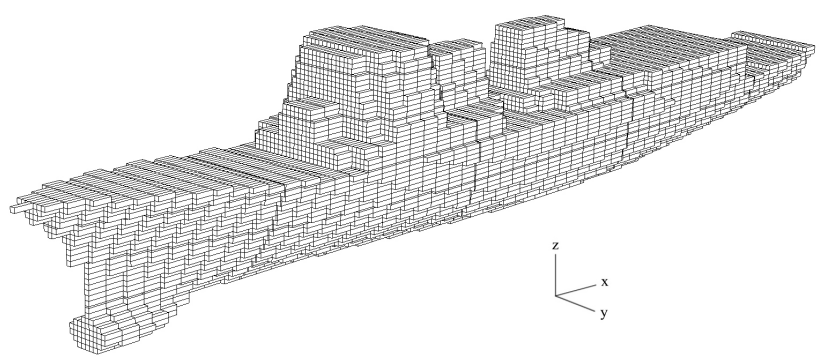

Figure 12: Final VEM ship mesh constituted of 33,364 VEs 


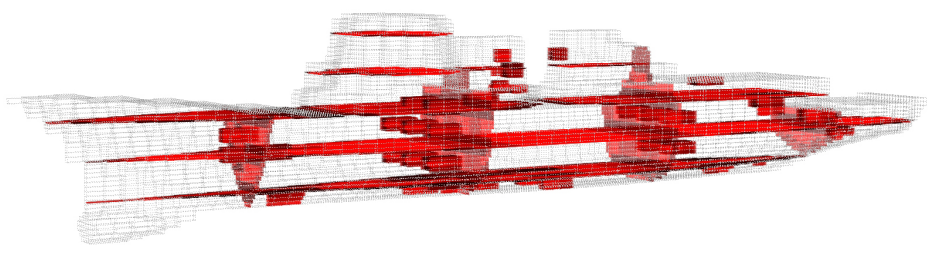

Figure 13: Components distribution within the notional all-electric ship comprising 43 equipment, four bulkheads, and five decks

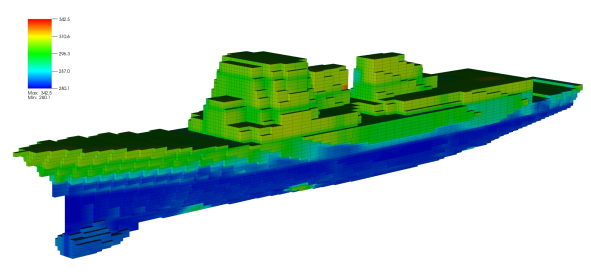

(a) Temperature field

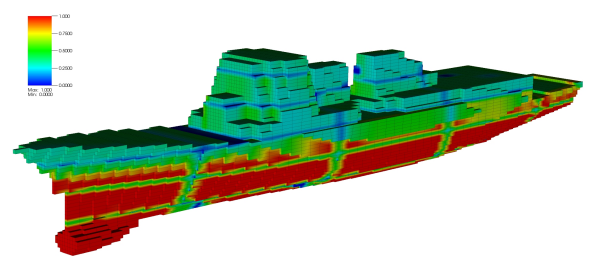

(b) Relative humidity field

Figure 14: Thermal response of a notional all-electric ship boundary

imposed on the top ship boundary increases the superstructure temperature. Red spot in the ship superstructure indicates the presence of a heat generating equipment. Relative humidity depicted in Figure $14 \mathrm{~b}$ is computed according to Eq. (9).

Figure $15 \mathrm{a}$ and $15 \mathrm{~b}$ show temperature and relative humidity fields, respectively, at the ship cross-section in the x-z plane at 7,200 s. In Figure 15a bulkheads and decks included in the ship mesh prevent the heat flow and thus cause significant temperature difference between the compartments. Moreover, 
the dividers can be used to separate and identify different zones and compartments, allowing for the zonal or compartmental thermal analysis of the ship. Red spots in Figure 15a indicate presence of thermal loads and these are identified as blue dots in Figure $15 \mathrm{~b}$ since solid components, including bulkheads and decks, have relative humidity of zero.
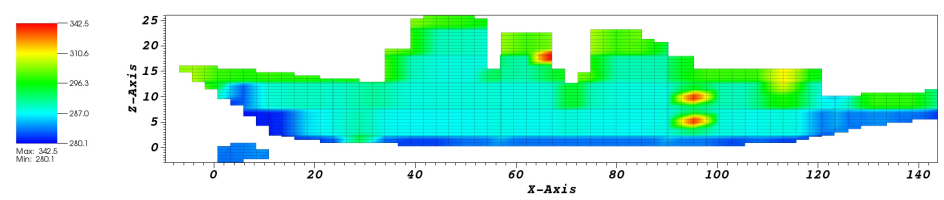

(a) Temperature field
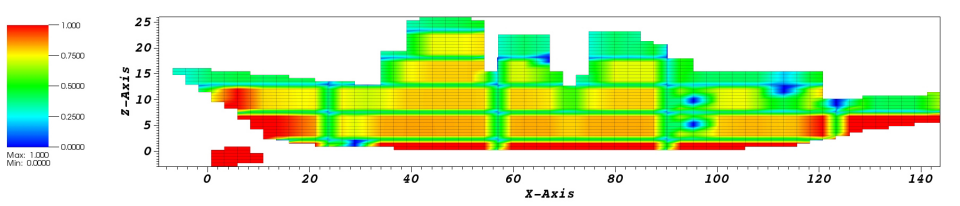

(b) Relative humidity field

Figure 15: Thermal response of a notional all-electric ship at its cross-section in the $\mathrm{x}-\mathrm{z}$ plane

\section{Conclusion}

360

VEM was presented with its application in ship thermal analysis. In order to construct meshes of large and complex ships with a small number of hexahedral elements, the present strategy adapted ray crossings and ray-triangle intersection algorithms developed in previous studies. In addition, the practicality of was illustrated by obtaining thermal responses of a notional all-electric ship comprising 43 thermal loads, four bulkheads, and five decks.

The final independent ship mesh was composed of 33,364 VEs built in 259.46 CPU seconds. In order to obtain ship temperature and relative humidity fields, laws of heat transfer and thermodynamics were applied to each VE along with empirical correlations, and the resulting system of nonlinear ODEs were solved 
with respect to time. Subsequently, ship temperature and relative humidity fields were visualized in VisIt software.

The following concluding remarks were construed from the mesh generation

\section{Acknowledgment}

This work was supported through the Office of Naval Research (ONR) grant N00014-14-1-0198.

1. The present VEM mesh generation strategy was able to produce sufficiently accurate hexahedral meshes of complex ship geometry. More accurate representation of the actual ship (e.g., smoother edges and curves) were achieved by increasing the number of VEs in each respective spatial direction. However, this number was directly proportional to the computational cost of the mesh generation process (see Table 3). Therefore mesh independence test, such as the one presented in this paper, must be performed to find the optimal number of VEs for mesh-independent numerical solution.

2. The VEM mesh generation strategy was demonstrated to play a key role in the enhancement of the VEM computational efficiency by producing a coarse independent mesh of a notional all-electric ship with intricate geometric features and multiple components using a small number of elements. Consequently, the VEM was capable of obtaining plausible ship thermal solutions with respect to time using such a mesh, requiring low computational cost in overall.

In summary, the VEM mesh generation strategy was verified as an efficient method for achieving sufficiently accurate ship representations and numerical solutions using a coarse independent mesh. Similarly, this paper addressed remarkable roles of the mesh generation strategy in demonstrating the VEM as a prominent tool in ship thermal modeling and simulation. 
[1] E. Dilay, J. V. C. Vargas, J. A. Souza, J. C. Ordonez, S. Yang, A. B. Mariano, A volume element model (VEM) for energy systems engineering,

(4) International Journal of Energy Research 39 (1) (2015) 46-74. doi:10. 1002/er.3209.

[2] C. Fletcher, Computational techniques for fluid dynamics, 1991.

[3] J. Vargas, A. Mariano, D. Correa, J. Ordonez, The microalgae derived hydrogen process in compact photobioreactors, International Journal of Hydrogen Energy 39 (18) (2014) 9588-9598.

[4] S. Yang, J. V. Vargas, T. Nunes, J. C. Ordonez, Development and implementation of a dynamic vapor compression refrigeration model into vemesrdc ship thermal management tool, in: Electric Ship Technologies Symposium (ESTS), 2015 IEEE, IEEE, 2015.

[5] E. Dilay, J. Vargas, J. Ordonez, S. Yang, R. Schrattenecker, M. Coleman, T. Chiocchio, J. Chalfant, C. Chryssostomidis, The experimental validation of a transient power electronic building block (PEBB) mathematical model, Applied Thermal Engineering 60 (1) (2013) 411-422.

[6] J. Ordonez, S. Yang, C. Ordonez, J. Vargas, T. Solano, M. Bublitz, E. Collins, Thermal simulation of an off-grid zero emissions building, in: ASME 2014 8th International Conference on Energy Sustainability collocated with the ASME 2014 12th International Conference on Fuel Cell Science, Engineering and Technology, American Society of Mechanical Engineers, 2014, pp. V002T06A005-V002T06A005.

[7] H. Childs, E. Brugger, B. Whitlock, J. Meredith, S. Ahern, D. Pugmire, K. Biagas, M. Miller, C. Harrison, G. H. Weber, H. Krishnan, T. Fogal, A. Sanderson, C. Garth, E. W. Bethel, D. Camp, O. Rübel, M. Durant, J. M. Favre, P. Navrátil, VisIt: An End-User Tool For Visualizing and 
Analyzing Very Large Data, in: High Performance Visualization-Enabling Extreme-Scale Scientific Insight, 2012, pp. 357-372.

[8] R. Schneiders, A grid-based algorithm for the generation of hexahedral element meshes, Engineering with computers 12 (3-4) (1996) 168-177.

[9] Y. Lee, D. Yang, Development of a grid-based mesh generation technique and its application to remeshing during the finite element simulation of a metal forming process, Engineering Computations 16 (3) (1999) 316-339.

[10] D.-Y. Kwak, Y.-T. Im, Remeshing for metal forming simulationspart ii: Three-dimensional hexahedral mesh generation, International Journal for Numerical Methods in Engineering 53 (11) (2002) 2501-2528.

[11] D.-Y. Kwak, Y.-T. Im, Hexahedral mesh generation for remeshing in threedimensional metal forming analyses, Journal of materials processing technology 138 (1) (2003) 531-537.

[12] J. Wang, Z. Yu, Feature-sensitive tetrahedral mesh generation with guaranteed quality, Computer-Aided Design 44 (5) (2012) 400-412.

[13] Y. Ito, A. M. Shih, B. K. Soni, Octree-based reasonable-quality hexahedral mesh generation using a new set of refinement templates, International Journal for Numerical Methods in Engineering 77 (13) (2009) 1809-1833.

[14] G. Legrain, R. Allais, P. Cartraud, On the use of the extended finite element method with quadtree/octree meshes, International Journal for Numerical Methods in Engineering 86 (6) (2011) 717-743.

[15] Z. Yin, Direct generation of extended STL file from unorganized point data, Computer-Aided Design 43 (6) (2011) 699-706.

[16] E. Ruiz-Gironés, X. Roca, J. Sarrate, The receding front method applied to hexahedral mesh generation of exterior domains, Engineering with Computers 28 (4) (2012) 391-408. 
[17] G. De Santis, M. De Beule, K. Van Canneyt, P. Segers, P. Verdonck, B. Verhegghe, Full-hexahedral structured meshing for image-based computational vascular modeling, Medical engineering \& physics 33 (10) (2011) 1318-1325.

[18] J. F. Shepherd, C. R. Johnson, Hexahedral mesh generation constraints, Engineering with Computers 24 (3) (2008) 195-213.

[19] A. Cifuentes, A. Kalbag, A performance study of tetrahedral and hexahedral elements in 3-D finite element structural analysis, Finite Elements in Analysis and Design 12 (3) (1992) 313-318.

[20] V. I. Weingarten, The controversy over hex or tet meshing, Machine design 66 (8) (1994) 74-76.

[21] S. E. Benzley, E. Perry, K. Merkley, B. Clark, G. Sjaardama, A comparison of all hexagonal and all tetrahedral finite element meshes for elastic and elasto-plastic analysis, in: Proceedings, 4th International Meshing Roundtable, Vol. 17, Sandia National Laboratories Albuquerque, NM, 1995, pp. 179-191.

[22] S. Vinchurkar, P. W. Longest, Evaluation of hexahedral, prismatic and hybrid mesh styles for simulating respiratory aerosol dynamics, Computers \& Fluids 37 (3) (2008) 317-331.

[23] S. Prakash, C. R. Ethier, Requirements for mesh resolution in 3D computational hemodynamics, Journal of biomechanical engineering 123 (2) (2001) $134-144$.

[24] O. Zienkiewicz, D. Phillips, An automatic mesh generation scheme for plane 475 and curved surfaces by isoparametricco-ordinates, International Journal for Numerical Methods in Engineering 3 (4) (1971) 519-528.

[25] I.-I. Kim, A development of data structure and mesh generation algorithm for whole ship analysis modeling system, Advances in Engineering Software 37 (2) (2006) 85-96. 
[35] P. J. Roache, K. N. Ghia, F. M. White, Editorial policy statement on the control of numerical accuracy, Journal of Fluids Engineering 108 (1) (1986) $2-2$.

[36] P. Frey, P.-L. George, Mesh generation, Vol. 32, John Wiley \& Sons, 2010. 